\title{
Tangence
}

\section{De Victor Hugo à Jacques Ferron : les parcours d'une interaction}

\section{Catherine Lamy}

Numéro 41, octobre 1993

Interdiscurtivité dans l'œuvre de Victor-Lévy Beaulieu

URI : https://id.erudit.org/iderudit/025779ar

DOI : https://doi.org/10.7202/025779ar

Aller au sommaire du numéro

Éditeur(s)

Tangence

ISSN

0226-9554 (imprimé)

1710-0305 (numérique)

Découvrir la revue

Citer cet article

Lamy, C. (1993). De Victor Hugo à Jacques Ferron : les parcours d'une

interaction. Tangence, (41), 86-94. https://doi.org/10.7202/025779ar d'utilisation que vous pouvez consulter en ligne.

https://apropos.erudit.org/fr/usagers/politique-dutilisation/ 


\title{
De Victor Hugo à Jacques Ferron: les parcours d'une interaction \\ Catherine Lamy*
}

\begin{abstract}
Car un livre véritable, par-delà toutes les anecdotes qui le constituent, c'est d'abord la grande expérience d'écriture qui le détermine.
\end{abstract}

Victor-Lévy Beaulieu, Docteur Ferron

Si deux séries identifiées comme telles par leur auteur, soit Les voyageries et La vraie saga des Beauchemin, se sont développées - et se développent toujours dans le cas de la seconde dans les tours et détours de l'œuvre de Victor-Lévy Beaulieu, force nous est de remarquer que peu à peu et parallèlement à celles-ci, une autre série y prend elle aussi forme, celle-là apparemment à l'insu de l'écrivain. De Pour saluer Victor Hugo à Docteur Ferron, je crois en effet pouvoir avancer - c'est du moins le postulat premier de mes recherches - que s'est progressivement dessiné un trajet d'écriture tout à fait original dont les stations intermédiaires seraient Jack Kérouac et Monsieur Melville ${ }^{1}$. L'un après l'autre, ces quatre textes, à la fois essais, biographies, romans et peut-être surtout autobiographies, à la fois semblables et tout à fait différents, tracent dans l'œeuvre de Beaulieu un troisième et remarquable sillon qui n'est qu'en apparence diffus. Postulant que ces quatre titres construisent déjà un discours théoriquement complet, j'omets volontairement le tout récent Seigneur Léon Tolstoï/Sopbie et Léon ${ }^{2}$ constituant par ailleurs à mon sens sinon le "véritable" hommage de Beaulieu à

\footnotetext{
* Catherine Lamy est membre du Centre de recherche en littérature québécoise et étudiante à l'Université Laval.

1 Victor-Lévy Beaulieu, Pour saluer Victor Hugo, Montréal, Stanké, coll. "10/10", 1971; Jack Kérouac, Montréal, Stanké, coll. "10/10", 1973; Monsieur Melville, Montréal, VLB éditeur, 1978 et Docteur Ferron, Montréal, Stanké, 1991.

2 Víctor-Lévy Beaulieu, Seigneur Léon Tolstoï/Sopbie et Léon, Montréal, Stanké, 1992.
} 
Tolstoï, du moins l'ouvrage qu'il faudrait voir et mettre en rapport et en continuité avec Docteur Ferron.

Du salut à Victor Hugo au "pèlerinage" aux pays de Jacques Ferron, c'est un apprentissage, une compréhension toujours plus lucide et plus explicite de l'écriture et de la fiction, qui nous sont donnés à lire. Scandant plus de vingt ans d'écriture - le Victor Hugo paraît en 1970, le Ferron en 1991 -, les quatre essais représentent les différents jalons de la constitution progressive d'une fictionnalisation "idéale" de l'interaction Beaulieu-Autre. Ils instaurent, suspendent puis finalement réduisent la tension de deux écritures, celle de l'élève et celle du maître, resserrée jusqu'à son aboutissement: la souveraine poésie. D'un texte à l'autre, le lecteur est témoin de l'approfondissement d'un même travail d'écriture; il est convié à la poursuite du parcours d'une même interaction.

Une première lecture des quatre ouvrages suffit pour constater que si le Victor Hugo et le Kérouac repoussaient déjà, jusqu'à les faire éclater, les frontières génériques, juxtaposant les discours autobiographique, biographique et historique, le Melville et le Ferron vont encore plus loin en fusionnant ces discours, leur univers ayant littéralement "basculé du côté de la fiction". Lorsque, relayant Lévy, Abel Beauchemin s'impose comme narrateur du Melville, il entraîne par le fictif de sa parole la réalité à se fondre dans la fiction pour devenir un des matériaux de son élaboration. Comme la trilogie avait regénéré l'écriture d'Abel qui s'essoufflait après les trois premiers tomes des Voyageries ${ }^{3}$ et relancé le cycle jusqu'à sa conclusion, elle résout ici par et dans la fiction les tensions de l'interaction écriture/lecture posée dès le Victor Hugo, et assure le passage de l'"essai-poulet " au "pèlerinage".

C'est donc la "lecture-fiction" de Monsieur Melville qui apparaît comme le point tournant de la recherche d'un équilibre

Sur cette question du caractère déterminant de Monsieur Melville dans le paysage des Voyageries, voir Louise Milot, "Les voyageries de Victor-Lévy Beaulieu: un voyage dans la fiction", Littérature québécoise; voix d'un peuple, voies d'une autonomie, Bruxelles, Éditions de l'Université de Bruxelles, 1985, p. 103-117 [édité par Gilles Dorion et Marcel Voisin]. Au fil d'une démonstration qui s'appuie sur les titres et sous-titres des six tomes du cycle, l'auteure de l'article met en lumière que de l'appareil titulaire du Melville, au seuil même du texte de Beaulieu, peut-être dégagée "une conception de la fiction qui lie, en logique et en simultanéité, la production de nouveaux objets fictifs d'écriture et la filiation par rapport à des sujets réels déjà existants" (p. 110). 
entre le déjà inventé et sa réinvention, le déjà lu/écrit et sa relecture/réécriture, que sous-tendent les quatre essais. Tant le fait qu'il ait justement fallu trois volumes sur Melville, que l'inscription de l'ouvrage au cœur même des Voyageries, et jusqu'au caractère nécessaire d'un tel livre dans le déroulement du cycle romanesque mais aussi semble-t-il dans celui de La vraie saga des Beauchemin, le souffle de Moby Dick devant "transform[er] et arm[er Abel] comme il convient de l'être lorsqu'on veut écrire la Grande Tribu et tout ce qui pourrait encore survenir d'elle " ${ }^{4}$, tout cela laissait déjà présager que l'hommage à l'écrivain américain deviendrait aussi, dans la série des essais, un lieu clé. Pour parler selon les termes de la sémiotique greimassienne, en appliquant le schéma canonique de la narrativité au discours englobant que constituerait la mise en linéarité des énoncés des quatre ouvrages, la station du Melville - la troisième - occuperait la position centrale de la performance, tandis que le Victor Hugo, le Kérouac et le Ferron correspondraient respectivement aux phases de manipulation, de compétence et de sanction ${ }^{5}$.

Si les deux parcours, celui des Voyageries et celui des essais, ne paraissent se croiser de façon tangible qu'au lieu du Melville, leur interdépendance est à mon sens beaucoup plus profonde. Une confrontation des dates de parution nous apprend que la première série, qui se déploie de Blancbe forcée (1976) à Discours de Samm (1983), est en tout entière encadrée par la seconde, commencée en 1970 et bouclée en 1991 avec Docteur Ferron. De là à poser l'hypothèse que, bien au-delà de Monsieur Melville, c'est tout le cycle des Voyageries qui participe en sousmain à l'élaboration du discours construit par les quatre essais jusqu'à le mener "dans ses grosseurs", il n'y qu'un pas... qu'il est assez tentant de franchir. Comment en effet ne pas reconnaître par exemple dans la narration à trois voix - celle d'Abel bien sûr mais aussi celles de l'Amérindienne Samm et de Bélial, le "maître des enfers " devenu chauffeur particulier — du "pèlerinage" aux lieux de Ferron, l'alternance de la parole de Samm - "elle dit " et de l'écrit d'Abel - "il écrit" - de Discours de Samm, retravaillée par l'imaginaire de Beaulieu dans l'aura ferronnienne?

4 Victor-Lévy Beaulieu, Monsieur Melville, 1. Dans les aveilles de Moby Dick, p. 24.

5 Pour plus de détails sur une telle proposition théorique et pour une application comparable du schéma canonique, voir Louise Milot, op. cit. 
Comme la présence silencieuse de la Montagnaise, qui lisait dans la chambre mauve de Mattavinie les mots du Melville à peine tracés, s'était avérée essentielle à la poursuite de l'écriture d'Abel Beauchemin, c'est son discours qui rend littéralement possible le texte d'Abel dans le dernier volet des Voyageries et sa participation au voyage et à la narration de Docteur Ferron qui en garantit la mise en route, pourtant depuis longtemps projetée par Abel. Le parcours textuel de Samm transgresse ainsi les frontières des deux séries et suture, "par la fiction qui le détient", l'univers romanesque et l'entreprise d'essayiste de Beaulieu.

On remarque que déjà, de la prise en considération globale des textes, plusieurs pistes théoriques peuvent être soulevées autour d'une hypothèse majeure qui pose la suite des quatre essais comme un programme de redéfinition de la fiction. Au fil de l'écriture, l'imaginaire de Beaulieu met en place une interdiscursivité fictive, mais dont les relations réactivent nécessairement et de façon explicite en les retravaillant des pans de réalité. Reste maintenant à voir si la pertinence d'une telle observation peut être vérifiée par un travail sur le texte lui-même et surtout à démontrer comment, du niveau de l'énonciation, les différentes phases de cette fictionnalisation sont déportées vers celui de l'énoncé.

Il est assez évident que, dans les limites de cet article, le fragment d'analyse qui va suivre, loin de prétendre atteindre un objectif d'une telle envergure, cherchera plutôt à établir des correspondances et à dégager certains grands axes textuels, éventuellement vérifiés dans un second palier de travail. C'est dans une telle optique que je propose ici la mise en parallèle de deux extraits des segments initial et terminal de la série, Pour saluer Victor Hugo et Docteur Ferron. Tiré du premier chapitre de chacun des ouvrages, les deux passages mettent en scène ce qu'il est convenu d'appeler un "heureux événement": la (re)naissance de Lévy par la lecture des Misérables du "père "Hugo et la (re)naissance d'Abel par la celle, impromptue, de La nuit de Ferron. En toile de fond de l'argumentation, la similitude "anecdotique" des deux tableaux, qui appelait déjà leur mise en relation, permet de faire mieux ressortir comment, entre les deux occurrences, au lieu même du Melville, un déplacement s'est opéré. Le phénomène qui avait amené l'énonciateur du Victor Hugo et du Ferron à choisir des moyens tout à fait différents pour répondre à une même volonté, "saluer" une figure capitale du 
90

paysage de l'écrivain Beaulieu et de son double $\mathrm{Abel}^{6}$, est ainsi transposé dans l'énoncé des deux essais.

Dans toute la première partie de Pour saluer Victor Hugo ${ }^{7}$, les discours autobiographique et biographique sont tour à tour convoqués, s'interpellant l'un l'autre. Le "pacte autobiographique" est sauf: l'énonciateur, le narrateur et l'acteur principal correspondent bien à divers rôles assumés par un même "je", en l'occurrence celui de Victor-Lévy Beaulieu, suivant le niveau du texte où chacune des instances intervient. Par rapport au point de départ canonique du genre, l'origine du récit, au premier chapitre, paraît cependant déviée. Si l'on pouvait en effet s'attendre à ce que la narration démarre par la mise en scène de la petite enfance, sinon de la naissance du protagoniste, celui-ci nous est plutôt présenté au seuil de l'adolescence et d'une nouvelle vie à la ville, l'enfance à jamais traversée:

Ce sentiment [celui de la peur de la mort] n'avait fait que croitre depuis que nous étions déménagés à Rivière-des-Prairies, peutêtre parce j'avais été brutalement coupé de mon passé et qu'en faisant ce long voyage vers Morial, j'avais traversé une mort symbolique. Après cela, il n'y aurait plus jamais d'enfance, le connu deviendrait ce qui ne pourrait plus se vivre [...]. (p. 19)

Indiquant certes l'importance de la "cassure" provoquée par le déracinement familial mais aussi le caractère déterminant de l'époque dans l'existence à la fois pragmatique, cognitive et passionnelle du sujet ${ }^{8}$, des répétitions presque mot pour mot de l'incipit - "J'avais treize ans." (p. 11) - viennent scander à trois

6 Je fais ici évidemment référence au titre du premier ouvrage et à ce passage du "liminaire " de Docteur Ferron: "De toute façon, l'essentiel pour moi n'est pas là: par les mots que j'ai écrits dans Docteur Ferron, j'ai désiré saluer à ma manière le seul écrivain véritablement national que le Québec contemporain ait produit. Et j'ai désiré le saluer au moyen du pèlerinage [...]. (p. 12)

7 L'essai consacré à l'auteur des Misérables est en effet divisé en trois parties. La première entremêle autobiographie et biographie, la deuxième rend compte de l'accueil qu'a réservé le Québec à l'écrivain français, tandis que la troisième dresse une "anthologie arbitraire" de son oeuvre. Ne pourrait-on pas voir dans cette segmentation les prémisses de celle du Melville en trois tomes: Dans les aveilles de Moby Dick, Lorsque souffle Moby Dick et L'après Moby Dick ou la souveraine poésie?

8 Sur cette question de la triplication du niveau narratif, voir Algirdas Julien Greimas et Jacques Fontanille, Sémiotique des passions. Des états de choses aux états d'âme, Paris, Seuil, 1991. 
reprises le déploiement du chapitre: "Mais j'avais treize ans maintenant." (p. 12), "À treize ans, c'est un professeur [...]" (p. 13), "Treize ans donc" (p. 15). Entre la deuxième et la troisième inscription, l'investissement émotif des fameux "treize ans" s'est modifié, passant d'une première catégorisation dysphorique, puisqu'essentiellement liée à l'hostilité de la ville, à une seconde, celle-là euphorique ${ }^{9}$, car associée à la découverte de la littérature et surtout à la première lecture des Misérables de Victor Hugo. Le déplacement spatial des Beaulieu en recouvre donc un autre qui, grâce à l'heureuse initiative d'un professeur, entraîne Lévy, déjà lecteur assidu de $X-13$ et de l'Encyclopédie de la jeunesse, à pénétrer jusqui'à s'y perdre le monde de la "grande" littérature.

Avant le point tournant des "treize ans" et de la première visite à la Bibliothèque municipale, les seize volumes de l'Encyclopédie de Grolier sont la principale source de lecture du sujet ${ }^{10}$. Véritable "folerie" du père qui l'avait acheté au moment de la crise, l'ouvrage devient capital pour le fils: "Presque toute mon enfance est dans cette Encyclopédie [...]" (p. 12). Par l'achat du livre qui renferme ainsi l'"essentiel" de l'enfance de Lévy, le père amorce la redéfinition, ou plutôt le déplacement des contenus du terme même d' essentiel " de la dimension pragmatique, où ils correspondaient dans l'univers de la mère au gîte et à la nourriture, à la dimension cognitive. Relayant le geste du père, c'est à la parole d'Hugo qu'il revient de boucler et de valider la conversion: "J'abusai donc de cette phrase parce qu'elle m'était une nourriture" (p. 20, nous soulignons) ${ }^{11}$.

9 Appartenant au métalangage de la sémiotique greimassienne, les termes "euphorie" et "dysphorie" articulent la catégorie thymique "dont la dénomination est motivée par le sens du mot thymie - "humeur, disposition affective de base" (Petit Robert) [...]". (Algirdas Julien Greimas et Joseph Courtés, Sémiotique. Dictionnaire raisonné de la théorie du langage, I, Paris, Hachette-Université, 1979, p. 396).

10 Outre les illustrés - X-13, Albert Brien et Pit Vercbères -, il y avait aussi les prix de fin d'année, lus "régulièrement tous les trois mois "; Le bossu de Paul Féval, Le glaive de Cologne de Jean-Louis Foncine, Pieds nus dans l'aube de Félix Leclerc, La couleuvrine et un martyrologue romain.

11 Cette conception de la lecture comme nourriture est réactivée par Beaulieu au tout début du deuxième chapitre, mais à propos des "inoubliables romans" de Jean Giono: "tout se déroulant comme si [...] je lisais le roman de mon enfance, embelli mille fois par les belles phrases de Giono, savoureuses comme ces bons fruits que nous commençions [sic] enfin à manger. (p. 23, nous soulignons). 
92

Devant la passion que font naître chez Lévy l'épopée des Misérables et la découverte de la puissance esthétique de l'écriture, la figure du père ne peut qu'être submergée, elle aussi emportée par la démesure de Victor Hugo. Si modestement il appuyera toujours à sa façon l'entrée en écriture de son fils, le père devra céder au "maître " son rôle tutélaire. En juxtaposant plus tard à son prénom celui de l'écrivain, Victor-Lévy Beaulieu viendra a(e)ncrer dans l'espace et mettre littéralement au jour cette adoption inversée.

C'est donc bien par la narration de la naissance d'un sujet que commence Pour saluer Victor Hugo, mais d'un sujet à la fois cognitif et passionnel, "mis au monde" par la lecture initiatique des Misérables. Les "milliers de phrases" d'Hugo, qui parviennent à transmuer la laideur en beauté, agissent en manipulation et conduisent Lévy adolescent à écrire, "inlassablement et avec rage, d'énormes romans" (p. 23). L'écriture seule peut enrayer la mort symbolique des "treize ans"; elle trace un pont fictif entre l'enfance à Saint-Jean-de-Dieu et la nouvelle vie à Rivière-des-Prairies.

Cette fonction "thérapeutique" de la lecture et de l'écriture est réactivée de façon magistrale dans le passage retenu de Docteur Ferron, où Abel relate les circonstances de sa première lecture de La nuit (p. 22-23). Comme la "rencontre" de Victor Hugo avait transformé la "fracture" de la migration familiale jusqu'à en faire, pour Lévy, le lieu de la mise en route d'une aventure inespérée, celle de Ferron convertit la "blessure" d'Abel en ouverture vers le pouvoir à la fois fictif et réel de l'écriture. Au fil du déploiement syntagmatique de l'extrait, Abel est ainsi conduit d'un état initial où, "la maladie [1]'ayant traversé", il ne fait plus que lire, sans d'ailleurs réussir à le comprendre, tout ce qui lui tombe sous la main, à une situation qui le pose finalement comme ayant écrit un premier roman, Ti-Jean dans sa nuit. Entre les deux états: l'inscription de La nuit dont le narrateur lui-même atteint, non de poliomyélite mais de tuberculose, indique au patient Abel la voie de la guérison:

De cette première lecture de La nuit, c'est tout ce que j'ai retenu en 1965: que les mots peuvent être au-delà même de toute maladie. Et cette leçon de chose m'a fait écrire ce roman que j'ai intitulé Ti-Jean dans sa nuit et qui m'a valu d'être reçu, pour la première fois, chez un éditeur. (p. 23)

Le titre du quatrième essai de Beaulieu prend tout son sens: par la médiation de son écriture, Ferron agit bien en docteur auprès 
d'Abel malade ${ }^{12}$, en lui "ouvrant le pays". Au-delà même de la guérison, c'est une seconde naissance qui lui est alors offerte, comme le confirme Bélial dont la voix vient momentanément interrompre, pour mieux le relancer, le récit d'Abel: "Comme pour la première naissance d'Abel, j'étais là aussi" (p. 22). Si La nuit paraît ainsi avoir regénéré le sujet de l'énoncé ${ }^{13}$, il faut bien voir qu'au terme du "pèlerinage", une fois tous les pays de Ferron traversés, le sujet de l'énonciation sera lui aussi profondément renouvelé:

J'ai l'impression d'être un tout petit enfant, j'ai l'impression que je viens tout juste de sortir d'entre les cuisses de mon père, j'ai l'impression non de renaître mais de venir au monde pour la première fois [...]. (chapitre 20, p. 408)

De façon encore plus explicite que dans le cas de Victor Hugo, dans plusieurs passages de l'essai ${ }^{14}$, la figure de Jacques Ferron est associée à celle du père, désormais évacué du récit puisque relayé par Samm. Disjointes mais complémentaires dans le Victor Hugo, les actions du père et du "père littéraire" se fusionnent ici. Les deux univers, celui de la famille et celui de l'écriture, qui sous-tendent l'œuvre de Victor-Lévy Beaulieu en entier, sont subsumées dans la seule figure de Ferron.

Et, comme l'on pouvait s'y attendre, ce glissement, de la disjonction à la conjonction, est entamé par l'intervention de Monsieur Melville. C'est "enfermé dans la maison du Père" qu'Abel lance sa poursuite à la trace de Moby Dick, dont la possibilitế

12 Elle aussi " thérapeutique", la lecture du Ciel de Québec avait quant à elle regénéré Samm: "Je me suis retrouvée à l'hôpital et c'est là que j'ai finalement lu Le ciel de Québec. En refermant le livre, je me suis souvenue de toi et du pèlerinage que tu m'as jadis proposé de faire dans tous les pays de Jacques Ferron. Je suis prête aujourd'hui à t'accompagner * (p. 28).

13 Point tournant dans l'existence d'Abel-acteur, le roman de Ferron l'est aussi dans celle d'Abel-narrateur, puisqu'il suspend à Trois-Pistoles, le temps du rituel de sa relecture, la "voyagerie" de la "Cadillac blanche dont les grands ailerons sont lumineux" (chapitre 7, p. 137-153).

14 Je pense ici tout particulièrement aux deux épisodes du "baptême" d'Abel par Jacques Ferron: "Mon fils, je te baptise au nom de Papa Boss, de son fils et de Pierre Elliott Trudeau. Pour les siècles des siècles, moi Ferron je te dis: ne sois jamais un tueur de pères. Amen" (p. 26); "Vous voilà vice-président à vie du Parti Rhinocéros et moi je serai dorénavant pour vous l'Éminence de la Grande Corne. Que Bélial vous vienne en aide, mon fils!" (p. 241). 
94

même dépend de la bonne volonté de Charles. Impatient de voir le projet de La grande tribu mis en branle et pour qu'Abel puisse enfin, par le fil de l'écriture, venir à bout d'Herman Melville, le Père Beauchemin a en effet accepté de prendre l'existence de son fils en charge, comme jadis il avait réussi à préserver "le langage du crayon" et rendu littéralement possible le tracé de l'écriture, en obtenant de l'institutrice qu'Abel écrive de la main gauche:

[...] c'était de la main que tout venait, c'était dans la main que se trouvait le lieu du langage. (Monsieur Melville, 1, p. 59)

Et c'est par le langage qu'on accède au seuil, et c'est par le langage que, peut-être, l'on peut espérer aller au-delà, même dans ce langage - dans le lieu souverain de la parole secrète [...]. (p. 67)

Dans le Melville, on le voit, la figure du père paraît ainsi intimement liée à l'écriture d'Abel, alors qu'au tout début de son parcours, dans Pour saluer Victor Hugo, elle était plutôt associée aux lectures de Lévy et que dans Docteur Ferron, pénétrant encore plus avant l'espace cognitif d'Abel, elle s'inscrira dans la figure même de Jacques Ferron qui, elle, porte à la fois les deux mondes, de la lecture et de l'écriture.

Si l'observation d'un seul parcours figuratif ne peut évidemment pas suffire, et j'en suis bien consciente, à démontrer la pertinence de l'hypothèse qui pose les quatre essais comme correspondant aux quatre phases du schéma narratif canonique, elle m'apparaît cependant déjà mettre au jour l'écheveau de corrélations qui relie entre eux les hommages à Hugo, Kerouac, Melville et Ferron. En aval des intentions de Victor-Lévy Beaulieu, la (re)constitution de la série est justifiée en même temps qu'émerge un réseau de déplacements et de transformations qui, de station en station, construit, en l'orientant, un discours fictif qui reste encore à éclaircir. 